\title{
Study of the Effect of the Field Emission on the Breakdown Voltage Characteristic of Direct Current Nitrogen Microdischarges
}

\author{
M. Radmilović-RAdjenović* And B. Radjenović \\ Institute of Physics, University of Belgrade, Pregrevica 118, Belgrade, Serbia \\ (Received April 27, 2018; revised version March 26, 2019; in final form March 28, 2019)
}

\begin{abstract}
This paper reports on theoretical studies of the role of the field emission effects in direct current nitrogen discharges between the electrodes separated from $0.5 \mu \mathrm{m}$ up to $100 \mu \mathrm{m}$. The effect of the various parameters on the breakdown voltage curves and current densities was calculated by using a Breakdown Voltage and Current Density in Microgaps Calculator. The obtained results clearly show that the shape of the potential barrier depends on the gap size and the work function. The high electric fields generated in microgaps combined with the lowering of the potential barrier seen by the electrons in the cathode as an ion approaches lead to the onset of ion-enhanced field emissions and the lowering of the breakdown voltage. Therefore, electrical breakdown across $\mu \mathrm{m}$ gaps is initiated by the secondary emission processes instead of a gas avalanche process and occurs at voltages far below the minimum predicted by the standard scaling law. It was found that the gap size, the gas pressure, enhancement factor, and the effective yield affect both the breakdown voltage curves and the current density. Presented results provide better understanding of the electrical breakdown in microgaps.
\end{abstract}

DOI: 10.12693/APhysPolA.136.114

PACS/topics: field emission, enhancement factor, microdischarges, breakdown voltage

\section{Introduction}

Microdischarges are characterized by their small size dimensions and high gas pressures yielding nonequilibrium plasmas [1-5]. Microdischarges have a huge potential in a wide range of future applications like display or photonics technology, embedded sensors, sources of UV light, and surface treatments [6-9]. However, despite of their potential applications in many different technological domains, the understandings of various physical phenomena, like their non-equilibrium behavior at high pressure, effect of pressure on breakdown in different configurations, gas temperature inside the micrometric configurations still require an understanding of the physics governing the new small-scale discharges.

In direct current microdischarges formed between the microscale interelectrode separations, field emission can act as a significant source of primary electrons [10-12]. The generation of microplasmas usually involves breakdown of the gas, where electrons accelerated in a high electric field trigger avalanche ionization. It was found that the onset of field emission in direct current microdischarges with gap sizes of the order of a few $\mu \mathrm{m}$ can lead to the breakdown at voltages far less than predicted by the standard scaling law. Deviations from the standard scaling law observed in microgaps can be described and explained by the quantum mechanical theory of field emission from metals [13-18].

*corresponding author; e-mail: marija@ipb.ac.rs
In this paper we present results regarding properties of nitrogen microdischarges generated between the electrodes separated by 0.5 up to $100 \mu \mathrm{m}$. Calculations were carried out by using breakdown voltage and current density in microgaps calculator [19]. This interface calculates breakdown voltage and the current density of the field emission driven discharges based on the formulation provided in [20]. Calculation conditions also include the gas pressure between $10^{5}$ and $5 \times 10^{5} \mathrm{~Pa}$, enhancement factor from 10 to 40 , the effective yield from 0.08 up to 1 , and the work function in the range $1.0-5.0 \mathrm{eV}$. The analysis of the breakdown voltage curves and current densities allow us to estimate the role of the field emission effect in generation of discharges in microgaps.

\section{Theoretical background}

Field emission, also known as the Fowler-Nordheim (F-N) tunneling, is the process by which free electrons are emitted from the metal surface when strong electric field is applied $[21,22]$. In a metal, electrons are usually prevented from escaping by a potential barrier separating the Fermi level in the metal and the vacuum level as shown in Fig. 1. When the electric field generated near the surface is satisfactorily large, electrons can tunnel through the potential hill and escape the surface. The width of the barrier decreases with increasing the field and when it becomes thin enough, the probability for electrons to tunnel through the barrier becomes non negligible and a field emission current appears. The field emission current density $j_{F E}$, a part of the flux density $n$ of electrons incident on the barrier from inside the conductor, is governed by the equation [23]: 


$$
j_{F E}=e \int_{0}^{\infty} n(\delta) D(\delta, E) \mathrm{d} \delta,
$$

where $\delta$ is the fraction of the electron's energy that is associated with the component of momentum normal to the surface of the conductor, $D$ is the transmission coefficient of the barrier, $E$ is the electric field, and $e$ is the electron charge. For direct current (DC) fields the current density is expressed by the F-N equation [23]:

$$
\begin{aligned}
& I_{F E}^{D C}=\frac{1.54 \times 10^{-6} \exp \left(4.52 \Phi^{-0.5}\right)(\beta E)^{2} A_{\Xi}}{\Phi} \\
& \quad \times \exp \left(\frac{-6.53 \times 10^{9} \Phi^{1.5}}{\beta E}\right),
\end{aligned}
$$

where $A_{\Xi}$ is an effective area of the emitter, $\Phi$ is the work function of the material (expressed in $\mathrm{eV}$ ), and $\beta$ is the enhancement factor. The traditional F-N equation established for perfectly planar and smooth surfaces is not suitable for describing emission from rough, irregular surfaces arising in modern nanoscale electron sources. For that reason, enhancement factor $\beta$ is defined as the ratio of the local emitter field over the applied field introduced in order to take into account the surface irregularities. Field emission results are more conventionally shown as the so-called F-N plot [23]:

$$
\frac{\mathrm{d}\left(\log _{10} I_{F E}^{D C} / E^{2.5}\right)}{\mathrm{d}(1 / E)}=-\frac{2.84 \times 10^{9} \Phi^{1.5}}{\beta} .
$$

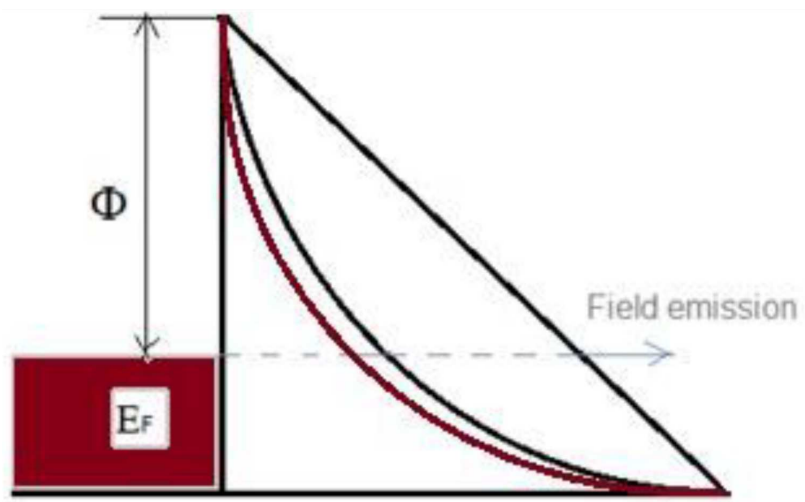

Fig. 1. Schematic diagram of the field emission in solids. $E_{F}$ is the Fermi level and $\Phi$ is the work function.

Another way to include the effect of surface irregularity on the electron tunneling is to introduce a fractionaldimension parameter $\alpha$. Then, the potential barrier is expressed as [24]:

$$
\begin{aligned}
& U_{b}(x)=-\frac{\hbar^{2}}{2 m_{e}}\left(\frac{\alpha-1}{2 x^{2}}+\frac{(\alpha-1)^{2}}{4 x^{2}}\right) \\
& +f^{2}(\alpha) x^{2 \alpha-2} V_{b}(x),
\end{aligned}
$$

where $f(\alpha)=\pi^{\alpha / 2} / \Gamma(\alpha / 2)$ and exact triangular barrier $V b(x)=E_{0}-e E x$, with $E_{0}=E_{F}+\Phi$.

\section{Results}

Figure 2 illustrates the shape of the potential barrier calculated in accordance with the expression (4) by using Mathematica [25] for $\Phi=4.6 \mathrm{eV}$ and $E_{F}=4.5 \mathrm{eV}$. For ideal planar surface, parameter $\alpha$ is equal to 1 , while $0<\alpha<1$ corresponds to reduced-dimensionality seen by the tunneling electrons. With decreasing parameter $\alpha$, the potential barrier is narrower and the probability of electron tunneling is higher. The potential barrier is much more affected by the parameter $\alpha$ than the work function as can be clearly noticeable from Fig. 3 .

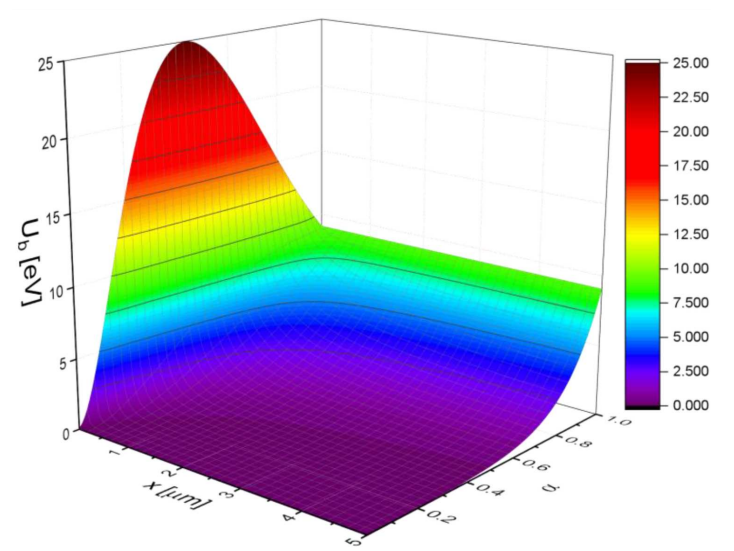

Fig. 2. The shape of the potential barrier versus the gap size and the parameter $\alpha$. Calculations were performed by using Mathematica [25] for $\Phi=4.6 \mathrm{eV}$ and $E_{F}=4.5 \mathrm{eV}$.

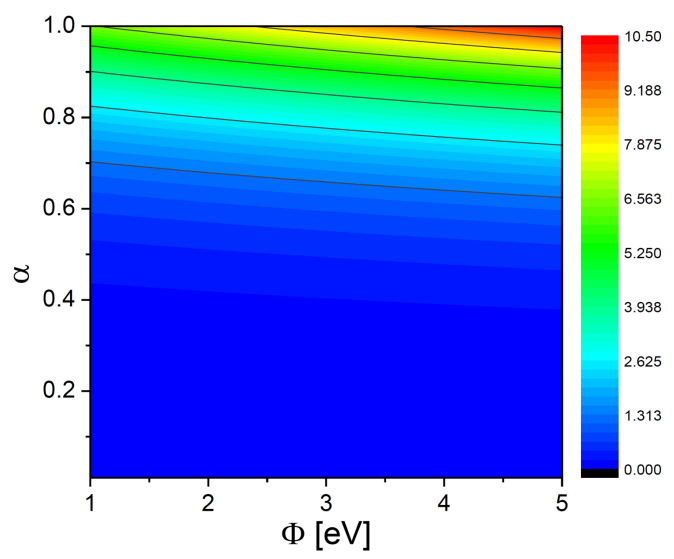

Fig. 3. The influence of the work function on the potential barrier for various fractional-dimension parametar $\alpha$. Calculations were performed by using Mathematica [25] for $E_{F}=4.5 \mathrm{eV}$ and the gap size of $2.5 \mu \mathrm{m}$.

The effect of the pressure on: (a) the breakdown voltage curve and (b) the current density is shown in Fig. 4. The pressure has strong influence on the right hand side of the breakdown voltage curves, while the left hand side is affected by the field emission. Electrons emitted from 
the surface due to strong electric field generated in microgaps lead to the lowering of the breakdown voltage and the departure from the standard scaling law. At larger gap sizes, the breakdown voltage and the current increase with increasing the gas pressure $[20,26]$.
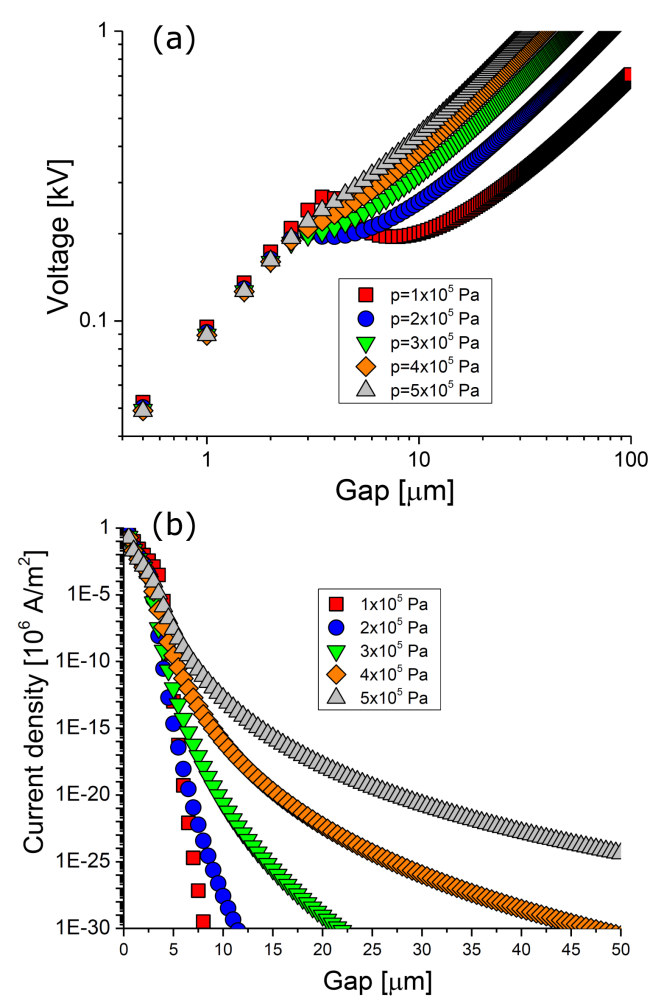

Fig. 4. (a) The breakdown voltage curves and (b) the current density as a function of the gap size and the voltage, for the gas pressure between $1 \times 10^{5} \mathrm{~Pa}$ and $5 \times$ $10^{5}$. Calculation conditions also include enhancement factor $\beta=5$, work function of $4.6 \mathrm{eV}$ and the effective yield of 0.1 .

The effect of the enhancement factor on: (a) the breakdown voltage curve and (b) the current density versus the gap size and the voltage is plotted in Fig. 5. As can be seen, the enhancement factor strongly affects the slope of the breakdown curve and the current density. Increase of the factor $\beta$ enhances the field emission and thereby decreases the breakdown voltage. For larger gap sizes, where field emission effects are negligible, there are no large differences among the breakdown voltage curves and current densities calculated for different values of the parameter $\beta$.

The strong influence of the secondary electron emission on: (a) the breakdown voltage and (b) the current density is demonstrated in Fig. 6. As expected, larger secondary emission causes the lowering of the breakdown voltage. However, at the gap sizes of a few $\mu \mathrm{m}$, the process of the electron emission due to the combined applied and ionic-space charge fields depends primarily on the electric field leading directly to the limitation of standard scaling law.
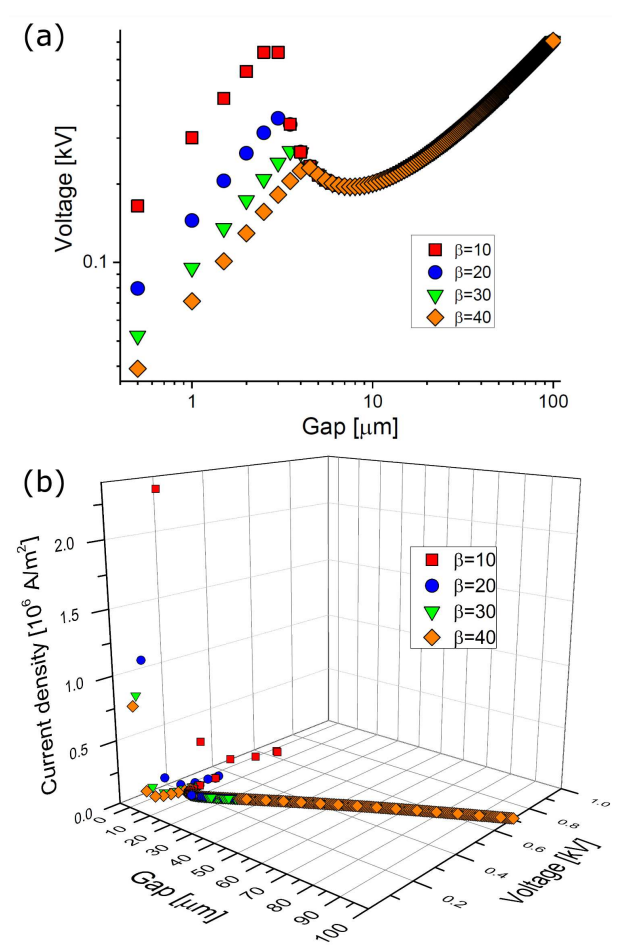

Fig. 5. The influence of the enhancement factor on: (a) the breakdown voltage curves and (b) the current density versus the gap spacing and the voltage. Calculations were performed for $\Phi=4.6 \mathrm{eV}$, the effective yield of 0.1 and the pressure of $1 \times 10^{5} \mathrm{~Pa}$.
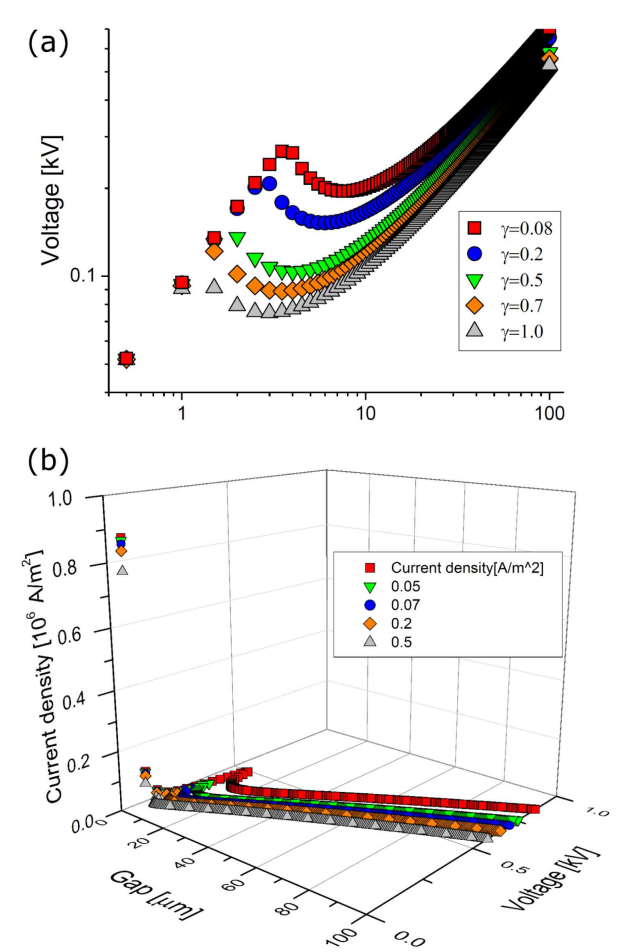

Fig. 6. (a) The breakdown voltage curves and (b) the current density against the gap size and the voltage calculated for various values of the effective yield, $\Phi=$ $4.6 \mathrm{eV}$, the pressure of $1 \times 10^{5} \mathrm{~Pa}$ and the enhancement factor $\beta=25$. 


\section{Conclusions}

This paper contains results of the theoretical studies of the breakdown mechanism in nitrogen microdischarges formed between 0.5 and $100 \mu \mathrm{m}$. The emphasis was put on the strong influence of the ion-enhanced field emission on the breakdown voltage curves and current densities in $\mu \mathrm{m}-$ and sub $\mu \mathrm{m}$-size gaps. Calculations were performed by breakdown voltage and current density in microgaps calculator [19] based on theory developed in [20]. It was shown that the field emission effect plays a significant role in the departure of the breakdown voltage from the standard scaling law within the range of high electric fields. As gap size is reduced, the electric field during breakdown becomes larger than the threshold for field emission causing a rapid reduction of the breakdown voltage. One possible mechanism responsible for the reduction of the breakdown voltage in microgaps is the enhancement of the secondary electron emission due to the quantum tunnelling of electrons from the metal electrodes to the gas phase. The width of the potential barrier depends on the gap size, the work function, and fractional-dimension parameter $\alpha$. As $\alpha$ decreases, the potential barrier becomes narrower and the probability of the tunneling becomes larger. The breakdown voltage curves and current density strongly depend on the gap size and the pressure. The enhancement factor is shown to be the most sensitive parameter with its increase leading to a significant drop in the threshold breakdown electric field and changes in the current density. The effective yield also affects both the breakdown voltage curves and the current density.

\section{Acknowledgments}

The present work has been supported by Ministry of Education, Science and Technology Republic of Serbia 171036 project.

\section{References}

[1] A. Shashurin, M. Keidar, Phys. Plasmas 22, 122002 (2015).

[2] K.H. Schoenbach, K. Becker, Eur. Phys. J. D 70, 29 (2016).

[3] A. Ligouri, A. Bigi, V. Colombo, M.L. Focarete, M. Gherardi, C. Gualandi, M.C. Oleari, S. Panzavolta, Sci. Rep. 6, 38542 (2016).

[4] A. Zeniou, N. Puač, N. Škoro, N. Selaković, P Dimitrakellis, E. Gogolides, Z.Lj. Petrović, J. Phys. D Appl. Phys. 50, 135204 (2017).
[5] V. Milosavljević, J. Vac. Sci. Technol. A Vac. Surf. Films 35, 03E105 (2017).

[6] S.J. Park, J. Chen, C. J. Wagner, N.P. Ostrom, C. Liu, J. G. Eden IEEE J. Select. Topics Quant. Electron. 8, 139 (2002).

[7] A. Saeed, A.W Khan, F. Jan, H.U. Shah, M. Abrar, M. Zaka-Ul-Islam, M. Khal, Plasma Sci. Technol. 16, 460 (2014).

[8] Y. Zhang, H. Y. Wang, Y. R. Zhang, A. Bogaerts, Plasma Sources Sci. Technol. 26, 054002 (2017).

[9] E. Tyczkowska-Sieron, R. Kapica, J. Markiewicz, J.Tyczkowsk, Plasma Med. 8, 57 (2018).

[10] D. Levko, L.L. Raja, J. Appl. Phys. 119, 163303 (2016).

[11] A. Nomin, A.V. Nominé, N.St.J. Braithwaite, T. Belmonte, G. Henrion, Phys. Rev. Appl. 8, 031001 (2017).

[12] M. Klas, L. Moravsky, ک̌. Matejčik, M. Zahoran, V. Martišovitš, B Radjenović, M RadmilovićRadjenović, Plasma Sources Sci. Technol. 26, 055023 (2017).

[13] M. Radmilović-Radjenović, B. Radjenović, Plasma Sources Sci. Technol. 17, 024005 (2008).

[14] D. Marić, N. Škoro, P.D. Maguire, C.M.O. Mahony, G. Malović and Z.Lj. Petrović, Plasma Sources Sci. Technol. 21, 035016(2012).

[15] M.U. Lee, J. Lee, J. K. Lee, G.S. Yun, Plasma Sources Sci. Technol. 26, 034003 (2017).

[16] G.N. Fursey, Field Emission in Vacuum Microelectronics, New York 2005.

[17] M. Radmilović-Radjenović, B. Radjenović, IEEE Trans. Plasma Sci. 35, 1223 (2007).

[18] D.B. Go, A. Venkattraman, J. Phys. D Appl. Phys. 47, 503001 (2014).

[19] K.S. Ahegbebu, Breakdown Voltage and Current Density in Microgaps Calculator, 2015.

[20] A. Venkattraman, A. Alexeenko, Phys. Plasmas 19, 123515 (2012).

[21] K.L. Jensen, Introduction to the Physics of Electron Emission, Wiley, Washington DC 2016.

[22] N. Egorov, E. Sheshin, Field Emission Electronics, Springer Int. Publ., Switzerland 2017.

[23] M. Klas, L. Moravsky, Š. Matejčik, B Radjenović, M Radmilović-Radjenović, IEEE Trans. Plasma Sci. 45, 906 (2017).

[24] M. Zubair, J.S. Ang, L.K. Ang, IEEE Trans. Electron Dev. 65, 2089 (2018).

[25] Wolfram Mathematica.

[26] M. Radmilovic-Radjenovic, B Radjenović, Š. Matejčik, M. Klas, Plasma Chem. Plasma Process 34, 55 (2014). 